\title{
Measuring the electron temperature by optical emission spectroscopy in two temperature plasmas at atmospheric pressure: A critical approach
}

\author{
A. Yanguas-Gil, a) J. Cotrino, and A. R. González-Elipe \\ Instituto de Ciencia de Materiales de Sevilla (CSIC-Univ. Sevilla), Dep. Física Atómica, Molecular y \\ Nuclear and Dep. Química Inorgánica (Univ. Sevilla), Av. Americo Vespucio 41092 Sevilla, Spain
}

(Received 11 March 2005; accepted 21 December 2005; published online 13 February 2006)

\begin{abstract}
The measurement of the electron mean kinetic energy by identifying the electron temperature and the excitation temperature obtained by optical emission spectroscopy is theoretically studied for two temperature argon plasmas at atmospheric pressure. Using a 32-level collisional radiative model in which both electron impact and argon-impact inelastic collisions are taken into account, it has been found that under certain conditions the argon inelastic collisions may cause a decrease of the argon excitation temperature so that the relation $T_{e}>T_{\text {exc }}>T_{0}$ is satisfied. This inequality also appears when electron losses due to diffusion are important and the electron density is lower than its equilibrium value. (C) 2006 American Institute of Physics. [DOI: 10.1063/1.2170416]
\end{abstract}

\section{INTRODUCTION}

Research on plasma discharges at atmospheric pressure has gained a renewed interest in the last years with the appearance of plasma devices in which, contrary to what happens with atmospheric arcs, the gas temperature is usually much colder than the electron mean kinetic energy. ${ }^{1-3}$

As the interpretation of the data obtained with electric probes at such pressures is not clear, ${ }^{4}$ many experimental works have focused on the use of optical emission spectroscopy (OES) as a way of characterizing the fundamental parameters of the discharge, either calculating the broadening of the emission lines to obtain the electron density, ${ }^{5}$ modeling emission bands of some species to get the gas temperature, ${ }^{6}$ or simply by recording the emission of the different excited states in order to get the electron temperature assuming a Bolztmann-like distribution of the population of the emitting excited levels. ${ }^{3}$

The determination of the electron mean kinetic energy through this method is based on the identification of the excitation temperature obtained with the electron temperature. ${ }^{7}$ This identification relies on the assumption of being in a situation close to the thermodynamic equilibrium in which all degrees of freedom share efficiently their energy. This approximation is supported by the high number of collisions that takes place at atmospheric pressure. The gas density is so high that the radiative processes are normally of second order compared to electron-impact processes in the kinetics of the excited states. Besides, self-absorption can play an important role in these conditions even for transitions between excited states, so that radiation trapping prevents the radiative decay to be an effective depopulation pathway of the excited states.

However, at such high densities, the excitation and deexcitation by collisions with neutral atoms must also be considered, since they can become one of the main loss pro-

${ }^{\text {a)} E l e c t r o n i c ~ m a i l: ~ a n g e l . y a n g u a s @ i c m s e . c s i c . e s ~}$ cesses for the excited states. ${ }^{8}$ Under the typical conditions of atmospheric arcs, the presence of these processes does not affect the populations of the excited states in a different way than that of the inelastic collisions by the electrons, as the gas temperature is normally very close to the electron temperature. ${ }^{9}$ The identification of electron temperature and excitation temperature for these discharges is therefore well justified. The problem arises when the gas is sensibly cooler than the electrons, as it is the case of some microwave discharges at atmospheric pressure. ${ }^{2,3}$ In these plasmas, the electron temperatures experimentally measured from the excitation temperature yield values that for the case of argon may be well below $0.4 \mathrm{eV}$, while the electron densities measured are over $10^{20} \mathrm{~m}^{-3}$. For these discharges, there are some experimental evidences that show that the excitation temperature can be higher than the electron temperature. ${ }^{10}$

In this work we have studied the relationship between electron temperature and excitation temperature in argon discharges at atmospheric pressure from a theoretical point of view, by using an argon collisional radiative (CR) model in which electron impact collisions, argon impact inelastic processes, and radiative decays are taken into account. The work is organized as follows: in Sec. II the CR model used and the processes considered are briefly described; the main results are presented and discussed in Sec. III and IV. Finally some conclusions are presented in Sec. V.

\section{ARGON COLLISIONAL-RADIATIVE MODEL}

The argon CR model developed comprises all the excited levels from argon ground state up to an excitation energy of $15 \mathrm{eV}$ so that, as it is shown in Table I, the behavior of 30 excited states can be followed.

In order to study the kinetics of the excited states at atmospheric pressure we have considered electron-impact, heavy particle-impact, and radiative processes.

For electron-impact inelastic processes, 
TABLE I. Summary of the argon excited levels considered.

\begin{tabular}{clcl}
\hline \hline Number & Configuration & Deg. & $\begin{array}{c}\text { Energy } \\
(\mathrm{eV})\end{array}$ \\
\hline 0 & $3 s^{2} p^{6}$ & 1 & 0 \\
1 & $4 s[3 / 2]_{2},{ }^{3} P_{2}$ & 5 & 11.548 \\
2 & $4 s[3 / 2]_{1},{ }^{3} P_{1}$ & 3 & 11.623 \\
3 & $4 s^{\prime}[1 / 2]_{0},{ }^{3} P_{0}$ & 1 & 11.723 \\
4 & $4 s^{\prime}[1 / 2]_{1},{ }^{1} P_{1}$ & 3 & 11.828 \\
$5-14$ & $4 p$ & 36 & $12.907-13.480$ \\
15 & $3 d 5 s$ & 48 & 14.019 \\
16 & $3 d^{\prime} 5 s^{\prime}$ & 24 & 14.246 \\
$17-26$ & $5 p$ & 36 & $14.464-14.738$ \\
27 & $4 d$ & 40 & 14.780 \\
28 & $6 s$ & 8 & 14.842 \\
29 & $4 f$ & 56 & 14.906 \\
30 & $4 d^{\prime}$ & 20 & 14.967 \\
31 & $3 s^{2} p^{5}$ & 6 & 15.76 \\
\hline \hline
\end{tabular}

- electron impact ionization and recombination to the ground state is as follows:

$e+\mathrm{Ar} \rightleftharpoons e+e+\mathrm{Ar}^{+}$

- electron impact ionization and recombination to an excited state is as follows:

$e+\mathrm{Ar}^{*} \rightleftharpoons e+e+\mathrm{Ar}^{+}$

- and electron impact excitation and deexcitation is as follows:

$e+\mathrm{Ar}^{*} \rightleftharpoons e+\mathrm{Ar}^{* *}$.

The electron-impact processes are characterized by a rate coefficient $K_{i j}$. For a direct transition $\left(\varepsilon_{j}>\varepsilon_{i}\right)$ this coefficient can be expressed as

$$
K_{i j}=\sqrt{\frac{2}{m}} \int_{\varepsilon_{i j}}^{\infty} \sigma_{i j}(\varepsilon) \varepsilon F(\varepsilon) d \varepsilon,
$$

where $\sigma_{i j}$ is the associated electron-impact cross section, $F(\varepsilon)$ the electron energy distribution function (EEDF) normalized so that $\int \varepsilon^{1 / 2} F(\varepsilon) d \varepsilon=1, \varepsilon_{i j}$ is the threshold energy, and $m$ the electron mass.

The rate coefficients for the inverse processes can be expressed by applying the detailed balance principle, ${ }^{11}$

$$
K_{j i}=\frac{g_{i}}{g_{j}} \sqrt{\frac{2}{m}} \int_{\varepsilon_{i j}}^{\infty} \sigma_{i j}(\varepsilon) \varepsilon F\left(\varepsilon-\varepsilon_{i j}\right) d \varepsilon
$$

and

$$
K_{c i}=\frac{g_{i}}{g_{e} g_{c}}\left(\frac{h^{2}}{2 \pi m k_{B} T_{e}}\right)^{3 / 2} \sqrt{\frac{2}{m}} \int_{\varepsilon_{i c}}^{\infty} \sigma_{i c}(\varepsilon) \varepsilon F\left(\varepsilon-\varepsilon_{i c}\right) d \varepsilon,
$$

with $g_{e}, g_{i}$, and $g_{c}$ the degeneracies of the electrons, the argon excited state, and the ionized fundamental state. $T_{e}$ is the electron kinetic temperature defined through $\langle u\rangle=\frac{3}{2} k_{B} T_{e}$, $\langle u\rangle$ being the mean kinetic energy and $k_{B}$ the Boltzmann's constant.

The atom impact processes considered are the following:
- atom impact ionization and three body recombination from the ground state,

$\mathrm{Ar}+\mathrm{Ar} \rightleftharpoons \mathrm{Ar}+e+\mathrm{Ar}^{+}$

- atom impact ionization and three body recombination from an excited state,

$\mathrm{Ar}+\mathrm{Ar}^{*} \rightleftharpoons \mathrm{Ar}+e+\mathrm{Ar}^{+}$

- and atom impact excitation and deexcitation,

$\mathrm{Ar}+\mathrm{Ar}^{*} \rightleftharpoons \mathrm{Ar}+\mathrm{Ar}^{* *}$

The neutral argon impact direct transitions are characterized by the rate coefficient,

$$
K_{i j}^{A}=\sqrt{\frac{2}{M}} \int_{\varepsilon_{i j}}^{\infty} \sigma_{i j}^{A}(\varepsilon) \varepsilon^{1 / 2} f^{A}(\varepsilon) d \varepsilon,
$$

$M$ being the argon mass, $\sigma_{i j}^{A}$ its associated cross section, and $f^{A}(\varepsilon)$ the neutral argon energy distribution function. The inverse processes rate coefficients are given again by simply applying the detailed balance principle, ${ }^{11}$

$$
K_{j i}^{A}=\frac{g_{i}}{g_{j}} \exp \left(\frac{\varepsilon_{i j}}{k_{B} T_{0}}\right) K_{i j}^{A},
$$

and

$$
K_{c i}^{A}=\frac{g_{i}}{g_{e} g_{c}}\left(\frac{h^{2}}{2 \pi m_{e} k T_{e}}\right)^{3 / 2} \exp \left(\frac{\varepsilon_{i c}}{k_{B} T_{0}}\right) K_{i c}^{A} .
$$

The radiative processes considered are the following:

- radiative recombination to the ground state,

$$
\mathrm{Ar}^{+}+e \rightarrow \mathrm{Ar}+h \nu
$$

- radiative recombination to an excited state,

$$
\mathrm{Ar}^{+}+e \rightarrow \mathrm{Ar}^{*}+h \nu
$$

- and radiative deexcitation,

$$
\mathrm{Ar}^{* *} \rightarrow \mathrm{Ar}^{*}+h \nu
$$

The radiative recombination rate coefficients can be expressed as ${ }^{11}$

$$
A_{c i}=\sqrt{\frac{2}{m}} \int_{0}^{\infty} \varepsilon^{1 / 2} q_{c i}(\varepsilon) f(\varepsilon) d \varepsilon,
$$

$q_{c i}$ being the radiative recombination cross section.

Taking into account the rate coefficients previously defined, the collisional balance equation for the excited state $i$ yields

$$
\begin{aligned}
\partial n_{i} /\left.\partial t\right|_{\mathrm{CR}}= & \sum_{j \neq i}\left(K_{j i} n_{e}+A_{j i}\right) n_{j}+K_{c i} n_{e}^{2} n^{+}+A_{c i} n_{e} n \\
& +\sum_{j \neq i} K_{j i}^{A}\left(n_{A}-n_{e}\right) n_{j}+K_{c i}^{A} n_{e} n^{+}\left(n_{A}-n_{e}\right) \\
& -\left(\sum_{j \neq i} K_{i j} n_{e}+\sum_{j \neq i} A_{i j}+K_{i c} n_{e}\right) n_{i} \\
& -\left(\sum_{j \neq i} K_{i j}^{A}\left(n_{A}-n_{e}\right)+K_{i c}^{A}\left(n_{A}-n_{e}\right)\right) n_{i},
\end{aligned}
$$

$n_{A}$ and $n_{e}$ being the gas and electron densities and $n^{+}$the 
TABLE II. Summary of references for the updated set of argon ground-state cross sections.

\begin{tabular}{|c|c|c|}
\hline Number & Configuration & Reference \\
\hline 1 & $4 s[3 / 2]_{2}$ & Khakoo et al. ${ }^{\mathrm{a}}$ \\
\hline 2 & $4 s[3 / 2]_{1}$ & Khakoo et al. ${ }^{\mathrm{a}}$ \\
\hline 3 & $4 s^{\prime}[1 / 2]_{0}$ & Khakoo et al. ${ }^{\mathrm{a}}$ \\
\hline 4 & $4 s^{\prime}[1 / 2]_{1}$ & Khakoo et al. ${ }^{\mathrm{a}}$ \\
\hline $5-14$ & $4 p$ & Chilton $e t a l .{ }^{\mathrm{b}}$ \\
\hline 15 & $3 d+5 s$ & Vlcek $^{\mathrm{c}}$ \\
\hline 16 & $3 d^{\prime}+5 s^{\prime}$ & Vlcek $^{\mathrm{c}}$ \\
\hline $17-26$ & $5 p$ & Weber et al. ${ }^{\mathrm{d}}$ \\
\hline 27 & $4 d$ & Drawin (1967) \\
\hline 28 & $6 s$ & Drawin (1967) \\
\hline 29 & $4 d^{\prime}$ & Drawin (1967) \\
\hline 31 & $3 s^{2} p^{5}$ & Rapp and Englander-Golden \\
\hline
\end{tabular}

${ }^{\mathrm{a}}$ Reference 20.

${ }^{\mathrm{b}}$ Reference 21.

${ }^{\mathrm{c}}$ Reference 11.

${ }^{\mathrm{d}}$ Reference 22.

${ }^{\mathrm{e}}$ Reference 23 .

density of Ar atomic ions. Losses due to diffusion have been considered just for the two metastable levels.

Finally, the contribution due to the $\mathrm{Ar}_{2}^{+}$species should be added to the previous equation. In the last years some works have appeared in which the role of molecular argon ion $\mathrm{Ar}_{2}^{+}$ has been taken into account at both low and atmospheric pressure discharges. ${ }^{8,12,13}$ The processes that lead to the formation and destruction of this species are well known as they are important in the modeling of argon excimer lasers. ${ }^{14}$ The presence of $\mathrm{Ar}_{2}^{+}$affects the excited states kinetics essentially in two ways: on one hand the three body recombination processes become less important as they are dependent on the population of atomic argon ions $\mathrm{Ar}^{+}$. On the other hand, the dissociative recombination reaction

$$
\mathrm{Ar}_{2}^{+}+e \rightarrow \mathrm{Ar}+\mathrm{Ar}(4 s)
$$

and the associative ionization

$$
\operatorname{Ar}(4 s)+\operatorname{Ar}(4 s) \rightarrow \mathrm{Ar}_{2}^{+}+e
$$

of the $4 s$ metastable levels affect the population of the $4 s$ excited levels.

For the electron impact processes, a complete set of cross sections has been recently compiled. ${ }^{15}$ This set is based not only in previous CR models available in literature, but also in the more recent experimental results, many of which have not been considered in previous collisional radiative models. ${ }^{11,12,16,17}$ In the case of electron impact excitation from the ground state, the validity of the cross sections used has been checked using an extended model by direct comparison with the transport coefficients and swarm data available in literature for argon. ${ }^{18}$

In Table II the set of argon inelastic cross sections from the ground state is presented. This set is based mainly in experimental measurements and has been validated by the comparison with the argon swarm data, and especially with the measurements of excitation coefficients of Tachibana. ${ }^{19}$ In order to get a good agreement, the measurements of Khakoo et al. ${ }^{20}$ for the argon metastable levels have been multi- plied by a factor 0.5 . For the upper excited levels for which there are no experimental data available, the Drawin formula for optically allowed transitions

$$
\sigma(u)=4 \pi a_{0} f_{i j}\left(\frac{E_{\mathrm{ion}}^{H}}{\varepsilon_{i j}}\right)^{2} \frac{(u-1)}{u^{2}} \log (1.25 u),
$$

where $a_{0}$ is the Bohr's radius, $E_{\text {ion }}^{H}$ the atomic hydrogen ionization energy, $\varepsilon_{i j}$ the threshold energy, $u=\varepsilon / \varepsilon_{i j}$, and $f_{i j}$ is the oscillator strength of the optical transition, has been used along with the oscillator strengths given by Lee and Lu. ${ }^{24}$ For inelastic processes between excited states, we have used the same cross sections presented in Yanguas-Gil et al.

The choice of the cross sections of the different argon inelastic collisions is of paramount importance in this work, as we are trying to study the influence of these proceses in the kinetics of the excited states. ${ }^{25}$ An extensive review of the published data was presented by Bultel et al. ${ }^{12}$ in 2002 and, as in their work, we have chosen the expressions given by Vlcek ${ }^{11}$ with the exception of the excitation from ground state to the $4 s$ configuration and the excitation exchanges between the $4 s$ levels. In these two cases the values listed in the work of Bultel et al. have been used.

Self-absorption has been introduced using the model developed by Holstein not only for resonant transitions but also for transitions between excited states. ${ }^{26-28}$ As it was previously mentioned, opacity in this discharges is very important, with escape factors for the $4 p-4 s$ transitions as low as $10^{-3}$.

\section{RESULTS}

The previous set of equations has been solved for conditions typical in microwave atmospheric discharges. Although the problem of calculating the electron energy distribution function in atmospheric pressure discharges has been tackled by some authors, ${ }^{29}$ this issue is beyond the scope of this work and instead we have used Maxwellian electron energy distribution functions for both the electrons and the neutral argon, an assumption which is reasonable in the case of the electrons due to the high electron density of these discharges. Therefore our model is characterized by the two temperatures $T_{e}$ and $T_{0}$. The excitation temperature has been determined from the slope of the populations of the excited states calculated by the model admitting that they follow the relation

$$
n_{i}=\frac{g_{i}}{g_{0}} \exp \left(\frac{-E_{i}}{k_{B} T_{\mathrm{exc}}}\right) .
$$

All the excited states from the $4 p$ excited configuration were considered, hence disregarding the contribution of the $4 s$ levels, which normally are not measured directly by OES, as the wavelengths of the transitions are well below $200 \mathrm{~nm}$.

In Fig. 1 the excitation temperatures obtained are presented assuming an equilibrium relation between the electron density and the electron temperature. When the heavy particle-inelastic collisions are neglected, the excitation temperature and the electron temperature have the same value except for low electron temperatures, in which the electron density is so low that the radiative processes become important and a corona model is obtained. In this regime the con- 


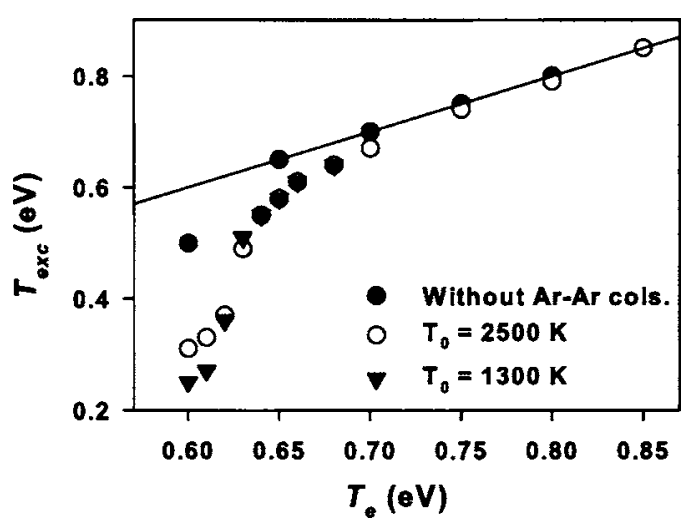

FIG. 1. Excitation temperatures obtained from the collisional radiative model for equilibrium electron densities. Results neglecting the contribution of the argon inelastic collisions (full circles) are compared with the complete two-temperature model for two gas temperatures, $T_{0}=1300 \mathrm{~K}$ (full triangles) and $T_{0}=2500 \mathrm{~K}$ (open circles). The line represents the $T_{\mathrm{exc}}=T_{e}$ reference.

cept of excitation temperature is meaningless due to the lack of interaction between the excited states. As the neutrals play no fundamental role in the kinetics of the excited states, a situation of thermodynamical equilibrium is reached when the electron temperature is high enough.

However, the introduction of the heavy-particle processes in the kinetic model causes a dramatic decrease in the excitation temperatures of the excited states presented in Fig. 1 for electron temperatures below $0.7 \mathrm{eV}$. As it is shown in Fig. 2 the argon inelastic processes play a net depopulating role in the kinetics of the argon excited states, hence reducing the excitation temperature by introducing a cooling effect that prevents the excited states from reaching the thermodynamical equilibrium with the electrons at low electron temperatures. When the electron temperature is increased the electron density also increases so that the electron collisional processes become more important and the system reaches the thermodynamic limit.

In Fig. 3 different excitation temperatures are presented for two constant values of electron density, $n_{e}=3$ $\times 10^{20} \mathrm{~m}^{-3}$ [Fig. 3(a)] and $n_{e}=8 \times 10^{20} \mathrm{~m}^{-3}$ [Fig. 3(b)]. In this case even when the argon inelastic collisions are neglected a lower value of the excitation temperature is found for high electron temperatures. As the electron density is

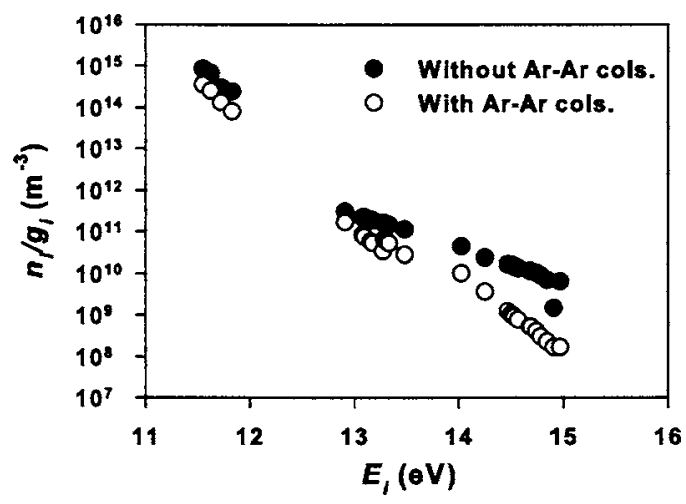

FIG. 2. Comparison of the populations of the excited states (ponderated by their degeneracies) both neglecting (full circles) and considering (open circles) argon-impact inelastic cross sections $\left(T_{e}=0.65 \mathrm{eV}, T_{0}=1300 \mathrm{~K}\right)$.
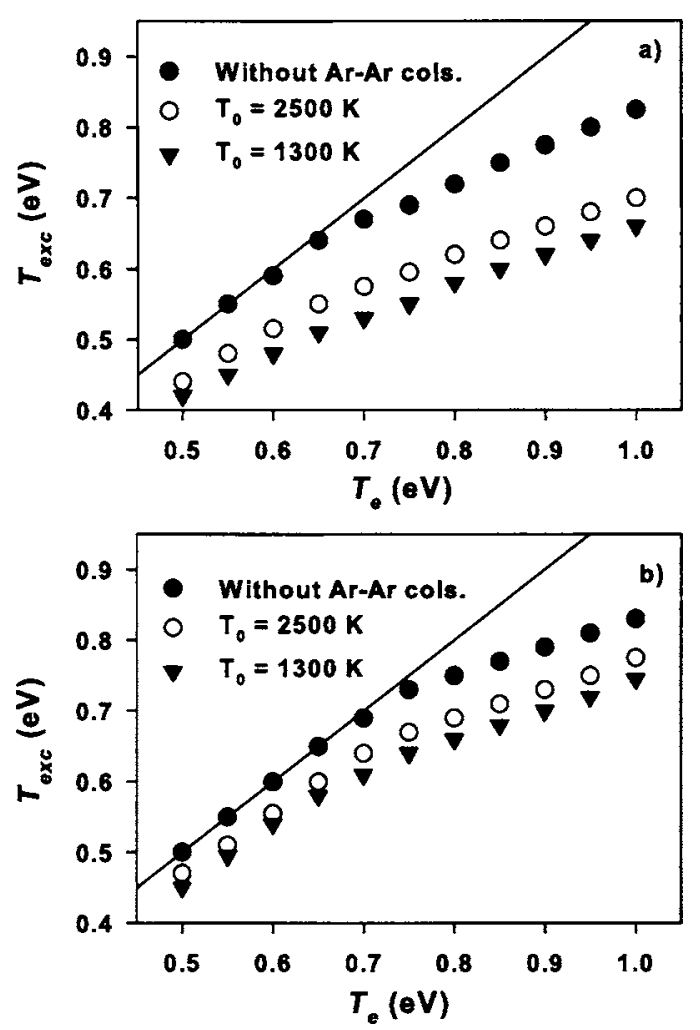

FIG. 3. Excitation temperatures obtained from the collisional radiative model considering two fixed values of the electron density: (a) $n_{e}=3$ $\times 10^{20} \mathrm{~m}^{-3}$ and (b) $n_{e}=8 \times 10^{20} \mathrm{~m}^{-3}$. Results neglecting the contribution of the argon inelastic collisions (full circles) are compared with the complete two-temperature model for two gas temperatures. The line represents the $T_{\mathrm{exc}}=T_{e}$ reference as in Fig. 1.

kept constant for all electron temperatures, the higher $T_{e}$ is the further is the electron density from its equilibrium value. Since the upper excited states are strongly linked with the ion and electron density due to the electron-impact ionization and recombination processes, the excited states try to reach the collisional balance with the electron density, so that the calculated excitation temperature yields values lower than the corresponding electron temperature. Note that from the experimental point of view, situations in which the electron density is lower than its equilibrium value have physical meaning so long as diffusive losses (i.e., wall losses) are important compared to the losses by three-body recombination. Therefore, according to our results the presence of these diffusive losses would also have a cooling effect in the argon excitation temperature. When the heavy particle inelastic processes are considered, the excitation temperature is even lower.

In Fig. 4 Boltzmann plots obtained for different $\mathrm{Ar}_{2}^{+}$concentrations show that the presence of this species affects greatly to the populations of the excited states. This mainly occurs due to the dissociative recombination reaction, which populates the $4 s$ levels.

However, as it can be seen in Fig. 4, variations on the population of the molecular ion $\mathrm{Ar}_{2}^{+}$do not affect greatly the excitation temperature. This is a consequence of the excitation exchanges between the different excited states, which are important enough to distribute effectively the population excess of the $4 s$ levels to the rest of Ar excited states. 


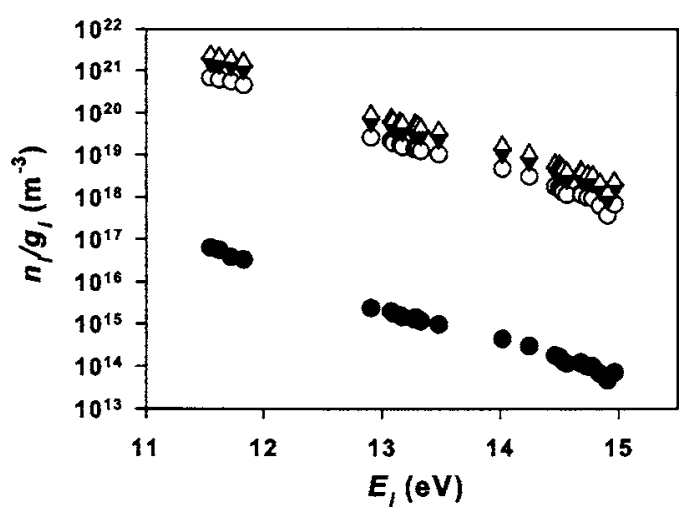

FIG. 4. Comparison of the populations of the excited states (ponderated by their degeneracies) for different values of the $\mathrm{Ar}_{2}^{+} / n_{e}$ ratio $\left(T_{e}=0.65 \mathrm{eV}\right.$, $n_{e}=6 \times 10^{20} \mathrm{~m}^{-3}$ ): $\mathrm{Ar}_{2}^{+} / n_{e}=0$ (full circles); $\mathrm{Ar}_{2}^{+} / n_{e}=0.2$ (open circles); $\mathrm{Ar}_{2}^{+} / n_{e}=0.5$ (full triangles); and $\mathrm{Ar}_{2}^{+} / n_{e}=0.9$ (open triangles).

\section{DISCUSSION}

The results presented in the previous section shows that even at atmospheric pressure under certain conditions the excitation temperature may differ from the electron temperature. Low electron temperatures, important differences between the electron and the gas temperature and departure of the electron density from the LTE condition, are the main factors that can reduce the excitation temperature.

When the electron temperatures are lower than approximately $0.7 \mathrm{eV}$, the atom impact processes act as a net depopulating pathway of the Ar excited states. This is a consequence of the detailed balance principle that relates excitation and deexcitation cross sections. In a twotemperature plasma, if the atom-impact excitation and deexcitation are of the same order of magnitude than the electron impact processes, there are two competing mechanisms, each of them involving particles which belong to ensembles characterized by different temperatures. Thus, the ensemble of the excited states will be characterized by an excitation temperature lower than the electron temperature. This effect is enhanced by the presence of low values of the electron temperature and density and high differences between the gas and the electron temperatures.

Important departures of the electron density from LTE values can also reduce the excitation temperature. In this case, the effect is caused by the strong interconnection between the Ar excited states and the $\mathrm{Ar}^{+}$ion. As the model assumes that ionization and recombination processes are related by the detailed balance principle, when $n_{e}$ is lower than the equilibrium value the net balance of ionization and recombination processes with the $\mathrm{Ar}^{+}$ion has a depopulating effect for the excited states, therefore reducing the excitation temperature of the ensemble. However, it must be noted that, contrary to the detailed balance principle for excitation and deexcitation processes, in which the relation was established for the cross sections, for electron-impact ionization and three-body recombination the detailed balance principle is an approximate relation that is strictly true just in the LTE situation. ${ }^{30}$

It is interesting also to check whether the results obtained may be of any use from the experimental point of
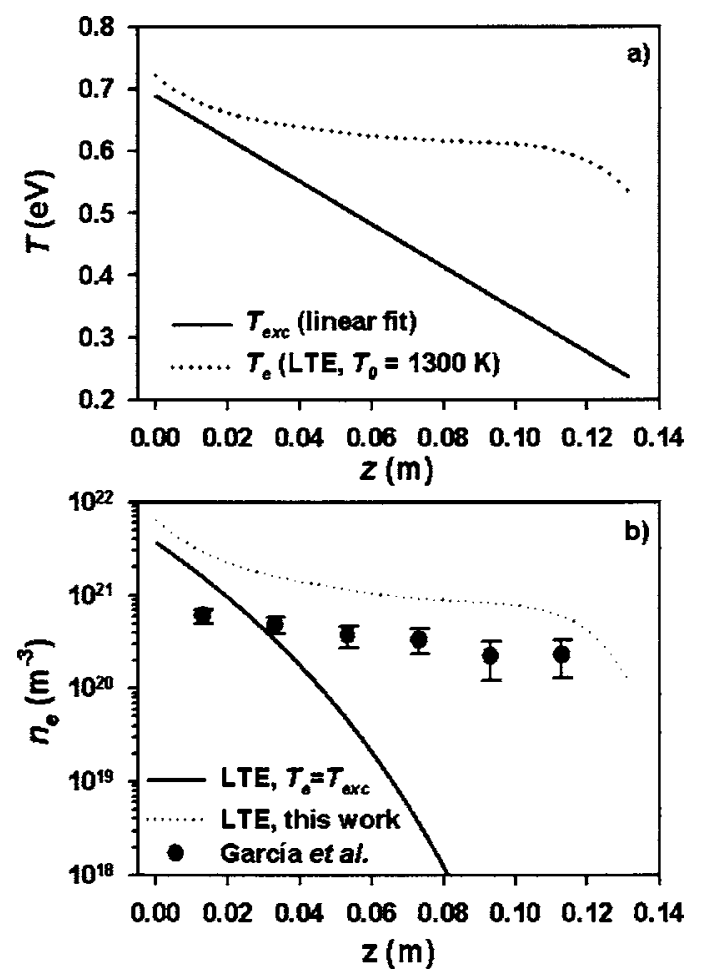

FIG. 5. Application of the model to a surface-wave discharge in argon at atmospheric pressure: (a) Comparison between the excitation temperature $T_{\text {exc }}$ measured by García et al. (Ref. 3) (linear fit) and the corresponding electron temperature $T_{e}$ according to the present model $\left(T_{0}=1300 \mathrm{~K}\right) ;(\mathrm{b})$ Comparison between the electron density $n_{e}$ experimentally measured by García et al. (Ref. 3) and the calculations using Saha equation for $T_{e}=T_{\text {exc }}$ and the electron temperature according to the present model $\left(T_{0}=1300 \mathrm{~K}\right)$.

view. As we have previously mentioned, differences between excitation and electron temperature are expected in situations in which gas temperature and electron density are low enough for the heavy-particle impact excitation and deexcitation to be important. According to the experimental results obtained by different authors, these conditions are often found in microwave discharges at atmospheric pressure. ${ }^{2,3}$

Thus, García et $a .^{3}$ obtained radially averaged measurements of the gas temperature, excitation temperature, and electron densities in an argon capillary surface-wave plasma at atmospheric pressure. Their axial results show electron densities in the range of $10^{-20} \mathrm{~m}^{-3}$, gas temperatures of $1300 \mathrm{~K}$, and excitation temperatures ranging from 0.7 to $0.3 \mathrm{eV}$. In Fig. 5(a), a comparison is presented between a linear fit of the excitation temperature measured by García et al. as a function of the axial position and the corresponding electron temperature obtained by the model described in this work for LTE conditions and a gas temperature of $T_{0}=1300 \mathrm{~K}$. The strong decrease of the excitation temperature experimentally obtained can be explained in terms of a slight decrease of the electron temperature, a situation which is congruent with the results obtained for this kind of plasmas at lower pressures. ${ }^{31}$

If we assume that the excitation temperature is nearly equal to the electron temperature, $T_{e} \approx T_{\text {exc }}$, strong differences appear between the electron densities experimentally measured by García et al. and the those obtained using the Saha equation [Fig. 5(b)], especially for higher values of $z$, 
where the experimental measurements are several orders of magnitude greater than the equilibrium densities. However, if we use the values of $T_{e}$ presented in Fig. 5(a), the values are always lower than the LTE values, but they follow the same axial profile. The differences would be explained through the presence of wall recombination processes for the charged species. These wall losses would reduce the electron density to values lower than those obtained through the Saha equation.

\section{CONCLUSIONS}

From this theoretical approach it can be concluded that even at atmospheric pressure there are certain conditions in which the measurement of the electron temperature by means of OES using Boltzmann plot methods may lead to an underestimation of the electron temperature, as the inequality $T_{e}>T_{\text {exc }}>T_{0}$ is satisfied. When the electron temperature is low enough, the argon inelastic collisions cause a decrease in the excitation temperature as calculated from the population of the upper excited states (i.e., from excited states whose radiative decays are in the UV/Visible (Vis) range) in two temperature discharges. The presence of diffusive losses (i.e., wall losses) can also cause important differences between excitation and electron temperatures in the discharge. These results could explain the low values of electron temperatures reported in some works on argon microwave discharges at atmospheric pressure. ${ }^{2,3}$

\section{ACKNOWLEDGMENTS}

This research was partially supported by Grant Nos. MAT2001-2820 and PPQ2001-3108 and the Acción Integrada HP02-102 from the Dirección General de Investigación Científica y Técnica (Spain).
${ }^{1}$ M. Moravej, X. Yang, G. R. Nowling, J. P. Chang, R. F. Hicks, and S. E. Babayan, J. Appl. Phys. 96, 7011 (2004).

${ }^{2}$ S. Y. Moon, W. Choe, H. S. Uhm, Y. S. Hwang, and J. J. Choi, Phys. Plasmas 9, 4045 (2002).

${ }^{3}$ M. C. García, A. Rodero, A. Sola, and A. Gamero, Spectrochim. Acta, Part B 55, 1733 (2000).

${ }^{4}$ M. C. T. Fang, J. L. Zhang, and J. D. Yan, IEEE Trans. Plasma Sci. 33, 1431 (2005).

${ }^{5}$ I. Holclajtner-Antunovic, M. Raskovic, and S. Jovicevic, Spectrochim. Acta, Part B 59, 419 (2004).

${ }^{6}$ C. de Izarra, J. Phys. D 33, 1697 (2000).

${ }^{7}$ E. Castanos-Martinez, Y. Kabouzi, K. Makasheva, and M. Moisan, Phys. Rev. E 70, 066406 (2004).

${ }^{8}$ A. Yanguas-Gil, J. Cotrino, and A. R. González-Elipe, Phys. Plasmas 11, 5497 (2004).

${ }^{9}$ A. B. Murphy, Phys. Rev. E 69, 016408 (2004).

${ }^{10}$ M. D. Calzada, M. C. García, J. M. Luque, and I. Santiago, J. Appl. Phys. 92, 2269 (2002).

${ }^{11}$ J. Vlcek, J. Phys. D 22, 623 (1989).

${ }^{12}$ A. Bultel, B. van Ootegem, A. Bourdon, and P. Vervisch, Phys. Rev. E 65, 046406 (2002).

${ }^{13}$ J. Jonkers, M. van de Sande, A. Sola, A. Gamero, A. Rodero, and J. van der Mullen, Plasma Sources Sci. Technol. 12, 324 (2003).

${ }^{14}$ S. Neeser, T. Kunz, and H. Langhoff, J. Phys. D 30, 1489 (1997).

${ }^{15}$ A. Yanguas-Gil, J. Cotrino, and A. R. González-Elipe, Phys. Rev. E 72, 016401 (2005).

${ }^{16}$ J. Bretagne, G. Callede, M. Legentil, and V. Puech, J. Phys. D 19, 761 (1986).

${ }^{17}$ A. Bogaerts, R. Gijbels, and J. Vlcek, J. Appl. Phys. 84, 121 (1998).

${ }^{18}$ A. Yanguas-Gil, J. Cotrino, and L. L. Alves, J. Phys. D 38, 1588 (2005).

${ }^{19}$ K. Tachibana, Phys. Rev. A 34, 1007 (1986).

${ }^{20}$ M. A. Khakoo et al., J. Phys. B 37, 247 (2004).

${ }^{21}$ J. E. Chilton, J. B. Boffard, R. S. Schappe, and C. C. Lin, Phys. Rev. A 57, 267 (1998).

${ }^{22}$ T. Weber, J. B. Boffard, and C. C. Lin, Phys. Rev. A 68, 032719 (2003).

${ }^{23}$ D. Rapp and P. Englander-Golden, J. Chem. Phys. 83, 1464 (1965).

${ }^{24}$ C. M. Lee and K. T. Lu, Phys. Rev. A 8, 1241 (1973).

${ }^{25}$ C. G. Braun and J. A. Kunc, Phys. Fluids 30, 499 (1987).

${ }^{26}$ T. Holstein, Phys. Rev. 72, 1212 (1947).

${ }^{27}$ T. Holstein, Phys. Rev. 83, 1159 (1951).

${ }^{28}$ P. J. Walsh, Phys. Rev. 116, 511 (1959).

${ }^{29}$ M. S. Benilov and G. V. Naidis, J. Phys. D 36, 1834 (2003).

${ }^{30}$ M. Mitchner and C. H. Kruger, Jr., Partially Ionized Gases (Wiley, New York, 1973).

${ }^{31}$ U. Kortshagen, A. Shivarova, E. Tatarova, and D. Zamfirov, J. Phys. D 27, 301 (1994). 